\title{
Combined approach to VHE gamma-ray astronomy at the TAIGA observatory
}

\author{
E. Postnikov* on behalf of the TAIGA collaboration \\ Skobeltsyn Institute of Nuclear Physics MSU, Moscow, Russia \\ E-mail: evgeny.postegmail.com
}

The TAIGA experiment is aiming to address important tasks in ground-based gamma-ray astronomy at energies from a few $\mathrm{TeV}$ to several PeV. TAIGA combines almost all the techniques for gamma-ray air shower detection:

1. First, an array of imaging air Cherenkov telescopes (one telescope already operating and two more to be added at the distance of 600-800 $\mathrm{m}$ from each other by 2019).

2. Second, an array of wide-angle Cherenkov timing detectors (currently 43 detector stations; up to 110 stations covering an area of $1 \mathrm{~km}^{2}$ in 2018).

3. Finally, an array of muon detectors covering a total area of $1 \mathrm{~km}^{2}$ with a detection area of $\sim 200 \mathrm{~m}^{2}$ by 2019 and up to $3000 \mathrm{~m}^{2}$ in the longer term.

Shower parameters are estimated using data of wide-angle Cherenkov timing detectors, whereas the selection of gamma-ray induced showers is based on the images of the telescopes taking into account shower parameters as well as muon array data.

Low investment coupled with high sensitivity $\left(2.5 \cdot 10^{-13} \mathrm{TeV} /\left(\mathrm{cm}^{2} \mathrm{sec}\right)\right.$ for $300 \mathrm{~h}$ of local sources observation at $100 \mathrm{TeV}, 1 \mathrm{~km}^{2}$ array) makes this pioneering approach very attractive for exploring the galactic PeVatrons.

The status and perspectives of the project as well as first results of the prototype phase are reported.

7th Fermi Symposium 2017

15-20 October 2017

Garmisch-Partenkirchen, Germany

\footnotetext{
* Speaker.
} 